\title{
Right Ventricular Dysfunction and Adverse Outcomes after Renal Transplantation
}

\author{
Megan S. Joseph ${ }^{a}$ Francis Tinney ${ }^{b}$ Abhijit Naikc Raviprasenna Parasuramanc \\ Milagros Samaniego-Picota ${ }^{d}$ Nicole M. Bhave ${ }^{a}$ \\ ${ }^{a}$ Division of Cardiovascular Medicine, Department of Internal Medicine, University of Michigan Medical School \\ and Michigan Medicine, Ann Arbor, MI, USA; 'D Department of Surgery, Henry Ford Health System, Detroit, MI, USA; \\ 'Division of Nephrology, Department of Internal Medicine, University of Michigan Medical School and Michigan \\ Medicine, Ann Arbor, MI, USA; dDivision of Nephrology, Department of Internal Medicine, Henry Ford Health \\ System, Detroit, MI, USA
}

\section{Keywords}

End-stage renal disease $\cdot$ Right ventricular dilation .

Right ventricular dysfunction · Renal transplant

\begin{abstract}
Introduction: Pulmonary hypertension is common among patients with end-stage renal disease, although data regarding the impact of right ventricular (RV) failure on postoperative outcomes remain limited. We hypothesized that echocardiographic findings of RV dilation and dysfunction are associated with adverse clinical outcomes after renal transplant. Methods: A retrospective review of adult renal transplant recipients at a single institution from January 2008 to June 2010 was conducted. Patients with transthoracic echocardiograms (TTEs) within 1 year leading up to transplant were included. The primary end point was a composite of delayed graft function, graft failure, and all-cause mortality. Results: Eighty patients were included. Mean follow-up time was 9.4 \pm 0.8 years. Eight patients (100\%) with qualitative RV dysfunction met the primary end point, while 39/65 patients (60.0\%) without RV dysfunction met the end point ( $p=$
\end{abstract}

karger@karger.com www.karger.com/crm

Karger $\stackrel{\text { ' }}{5}$
(C) 2021 The Author(s)

Published by S. Karger AG, Basel

This is an Open Access article licensed under the Creative Commons Attribution-NonCommercial-4.0 International License (CC BY-NC) (http://www.karger.com/Services/OpenAccessLicense), applicable to the online version of the article only. Usage and distribution for commercial purposes requires written permission.
0.026). Qualitative RV dilation was associated with a significantly shorter time to all-cause graft failure $(p=0.03)$ and death ( $p=0.048$ ). RV systolic pressure was not measurable in $45 / 80$ patients (56\%) and was not associated with outcomes in the remaining patients. Conclusion: RV dilation and dysfunction are associated with adverse outcomes after renal transplant. TTE assessment of RV size and function should be a standard part of the pre-kidney transplant cardiovascular risk assessment.

(C) 2021 The Author(s)

Published by S. Karger AG, Basel

\section{Introduction}

Cardiovascular disease is the leading cause of morbidity and mortality in patients with end-stage renal disease (ESRD) [1, 2]. Data from the United States Renal Data System have demonstrated that cardiovascular disease accounts for $31 \%$ of patient deaths with a functioning graft [3]. Much of the literature and clinical effort regarding preoperative risk stratification in this population focuses on ischemic heart disease and left-sided heart fail- 
ure $[1,2]$. The prevalence of pulmonary hypertension $(\mathrm{PH})$ in ESRD has been reported to be about 33\% [4], based on echocardiographic evidence of elevated right ventricular systolic pressure (RVSP). While elevated pretransplant RVSP has been associated with the adverse posttransplant outcomes in a number of studies [5], data regarding the impact of right ventricular (RV) failure on postoperative outcomes remain limited $[1,6]$.

Transthoracic echocardiography (TTE) is a widely available, relatively rapid, and very well-tolerated examination that is commonly used as a noninvasive screening tool for PH [7]. As outcomes in $\mathrm{PH}$ are closely linked to RV systolic function [8], assessment of RV size and function by TTE is invaluable for prognostication and management. We hypothesized that preoperative RV dilation and dysfunction as observed on TTE is associated with worse outcomes after renal transplantation, including delayed graft function, graft failure, and all-cause mortality.

\section{Materials and Methods}

\section{Study Population}

A retrospective review of adults receiving renal transplants at the University of Michigan from January 2008 to June 2010 was conducted. Patients who had TTEs, performed at our institution within 1 year prior to transplant were included in the analysis. Patients undergoing double organ transplant (heart-kidney, liverkidney, or kidney-pancreas) were excluded. The University of Michigan institutional review board approved the study. After transplantation, patients were treated with a standard triple immunosuppression protocol consisting of tacrolimus, mycophenolate mofetil, and maintenance-dose prednisone therapy. The primary end point was a composite of delayed graft function, graft failure, and all-cause mortality. Delayed graft function was defined as the need for hemodialysis within 1 week after transplantation.

\section{Clinical Data Collection}

Standard demographic and anthropometric data, laboratory test results, mode of dialysis, pretransplant medications, and outcomes data were collected from the electronic medical records. Clinical outcomes were extracted from the electronic medical records (Epic, Epic Systems Corporation, Madison, WI, USA) and from the University of Michigan Organ Transplant Information System (OTIS), which is a comprehensive transplant database. Preoperative hypotension was defined as systolic blood pressure $<90 \mathrm{~mm} \mathrm{Hg}$ at the final preoperative clinic appointment.

\section{Echocardiography}

Images were acquired using standard echocardiographic machines (Philips of Andover, MA, USA and Siemens of Washington, DC, USA). Complete TTEs with 2-dimensional imaging and Doppler evaluation were performed according to the standard clinical protocol. If multiple studies were obtained for a given patient within the year prior to transplantation, the study that occurred closest
Table 1. Baseline demographics of the study population $(N=80)$

\begin{tabular}{ll}
\hline Variable & Mean value or \\
patients, $n(\%)$
\end{tabular}

Age: mean $\pm \mathrm{SD}$, years

$51.3 \pm 14.2$

Gender

Male $\quad 58(72.5)$

Female 22 (27.5)

Primary etiology of renal failure

Diabetes mellitus $\quad 21(26.3)$

Hypertension $7(8.8)$

Multifactorial (DM \& HTN) $6(7.5)$

Glomerulonephritis $21(26.3)$

PCKD $8(10.0)$

Drug toxicity $7(8.8)$

Urologic issue $\quad 5(6.3)$

Other $5(6.3)$

Dialysis-dependent $\quad 62(77.5)$

On hemodialysis $\quad 54(67.5)$

On peritoneal dialysis $\quad 8(10)$

AV fistula present 39 (48.8)

Duration of dialysis, years: mean \pm SD $\quad 2.8 \pm 2.6$

Diabetes mellitus $33(41.3)$

Chronic systemic hypertension $68(85.0)$

Anemia 65 (81.3)

Type of renal transplant

Living unrelated donor $\quad 16(20.0)$

Living related donor $\quad 16(20.0)$

Deceased unrelated donor $\quad 48(60.0)$

Hypotension at the time of transplant $\quad 10(12.5)$

History of CAD 25 (31.3)

History of smoking $\quad 37$ (46.3)

DM, diabetes mellitus; HTN, hypertension; SD, standard deviation; PCKD, polycystic kidney disease; $\mathrm{AV}$, arteriovenous; CAD, coronary artery disease.

to the transplant was analyzed. All measurements were made by a blinded expert echocardiographer (NB). Standard linear measurements of the left ventricle (LV) and left atrium were made in the parasternal long-axis view. Left ventricular end-systolic and enddiastolic volumes were obtained using Simpson's method of discs, based on tracings in the apical 2-chamber and 4-chamber views. These volumes were used to calculate the biplane left ventricular ejection fraction (LVEF) as follows:

$\mathrm{LVEF}=[(\mathrm{LV}$ end-diastolic volume-LV end-systolic volume $) /$ LV end-diastolic volume] $\times 100 \%$ [9].

LVEF was also estimated visually. Left ventricular systolic dysfunction was defined as LVEF $<50 \%$, with calculated LVEF used preferentially over visually estimated LVEF, unless calculation was not possible due to limited image quality.

$\mathrm{RV}$ linear measurements were made in the apical 4-chamber view. As per the current guidelines, the upper limits of normal for these parameters are as follows: RV basal diameter, $41 \mathrm{~mm}$; RV mid-cavity diameter, $35 \mathrm{~mm}$; and RV length, $83 \mathrm{~mm}$ [10]. Qualitative RV dilation and dysfunction were adjudicated by the echocar- 
Table 2. Summary of echocardiographic findings

\begin{tabular}{|c|c|c|c|c|}
\hline \multicolumn{5}{|l|}{ LV size } \\
\hline $\operatorname{LVIDd}(\mathrm{mm} \pm \mathrm{SD})$ & $51 \pm 7.7$ & $51 \pm 8.0$ & $49 \pm 7.5$ & $2(2.5)$ \\
\hline \multicolumn{5}{|l|}{ RV size } \\
\hline $\mathrm{RV}$ basal diameter $(\mathrm{mm} \pm \mathrm{SD})$ & $39 \pm 7.3$ & $40 \pm 7.5$ & $38 \pm 6.8$ & $12(15)$ \\
\hline $\mathrm{RV}$ mid-cavity diameter $(\mathrm{mm} \pm \mathrm{SD})$ & $27 \pm 6.3$ & $27 \pm 7.0$ & $26 \pm 5.3$ & $22(27.5)$ \\
\hline $\mathrm{RV}$ length $(\mathrm{mm} \pm \mathrm{SD})$ & $76 \pm 9.2$ & $77 \pm 8.7$ & $75 \pm 9.9$ & $12(15)$ \\
\hline Qualitative RV dilation, $n(\%)$ & $16(20)$ & $13(25)$ & $3(10.7)$ & $5(6.3)$ \\
\hline \multicolumn{5}{|l|}{ RV function, $n(\%)$} \\
\hline $\mathrm{RA}$ area $\left(\mathrm{cm}^{2} \pm \mathrm{SD}\right)$ & $18 \pm 7.0$ & $18 \pm 6.2$ & $18 \pm 8.3$ & $10(12.5)$ \\
\hline $\operatorname{RVSP}(\mathrm{mm} \mathrm{Hg} \pm \mathrm{SD})$ & $31 \pm 9.0$ & $32 \pm 9.5$ & $30 \pm 9.5$ & $45(56.3)$ \\
\hline Moderate or greater tricuspid regurgitation, $n(\%)$ & $2(2.5)$ & $1(1.9)$ & $1(3.6)$ & $0(0)$ \\
\hline $\mathrm{E} / \mathrm{e}^{\prime} \geq 15, n(\%)$ & $7(8.8)$ & $6(11.5)^{9}$ & $1(3.6)$ & $19(23.8)$ \\
\hline
\end{tabular}

$\mathrm{SD}$, standard deviation; LVIDd, left ventricular internal diameter at end-diastole; LV, left ventricular; AP, antero-posterior; RV, right ventricular; LA, left atrial; RA, right atrial; RVSP, right ventricular systolic pressure. ${ }^{*} p=0.026$ for difference between patients who did and did not meet the primary end point. ${ }^{\dagger} p=0.486$ for difference. ${ }^{*} p=0.061$ for difference. ${ }^{*} p=0.10$ for difference. ${ }^{9} p=0.13$ for difference.

diographer based on a composite of the above variables and visual appearance of the RV.

Left atrial volume was obtained using Simpson's method of discs, based on tracing in the apical 4-chamber view. Right atrial area was traced in the apical 4 -chamber view and was considered to be abnormal if $>18 \mathrm{~cm}^{2}$ [9].

RVSP was estimated based on the peak tricuspid regurgitation velocity $(v)$ using the modified Bernoulli equation:

$\mathrm{RVSP}=4 v^{2}+$ estimated right atrial pressure [11].

Right atrial pressure was estimated based on inferior vena cava diameter and inspiratory collapsibility, as per the current guidelines. Left ventricular diastolic function was evaluated based on mitral valve inflow velocities (early or $\mathrm{E}$ wave, atrial or A wave, and E/A ratio), obtained using pulse-wave Doppler, and mitral annular tissue Doppler velocities (septal and lateral $e^{\prime}$, which were averaged).

\section{Statistical Analysis}

Continuous variables are reported as mean \pm standard deviation. Categorical variables are expressed as percentages. The difference between the patients meeting the primary end point and those who did not were assessed using an unpaired $t$ test for continuous variables and the $\chi^{2}$ test for categorical variables. KaplanMeier survival analyses were performed to assess time to all-cause graft failure and time to death. A correlation analysis was performed to assess the relationship between RVSP and RV basal di- ameter. Analyses were conducted using SPSS (IBM SPSS Statistics, Chicago, IL, USA) and Stata (StataCorp, College Station, TX, USA). The $p$ values reported are 2 -sided and considered significant at $p<0.05$.

\section{Results}

\section{Subject Characteristics}

A total of 80 adult kidney transplant patients were included in the study, with a mean follow-up time of $9.4 \pm$ 0.8 years. Baseline demographics of the study population are presented in Table 1 . The mean age of transplant recipients was $51.3 \pm 14.2$ years (range, $19-75$ years). The most common etiologies of renal failure were diabetes mellitus (21 patients, 26.3\%), glomerulonephritis (21 patients, $26.3 \%$ ), and polycystic kidney disease (8 patients, $10.0 \%)$. A majority of transplant recipients (62 patients, $77.5 \%$ ) were dialysis-dependent leading up to transplant, with the most common modality being hemodialysis (54 patients, $67.5 \%$ ). The mean duration of dialysis prior to transplant was $2.8 \pm 2.6$ years. Over the follow-up period, 
Table 3. Echocardiographic findings according to AV fistula status

\begin{tabular}{llll}
\hline Variable & \multicolumn{2}{l}{ Patients, $n(\%)$} & $p$ value \\
\cline { 2 - 3 } & $\begin{array}{l}\text { in patients with AV } \\
\text { fistulas }(n=39)\end{array}$ & $\begin{array}{l}\text { in patients without } \\
\text { AV fistulas }(n=41)\end{array}$ \\
\hline LV dilation $(n=13)$ & $6(46.2)$ & $7(53.8)$ & 0.90 \\
LV ejection fraction $<50 \%(n=9)$ & $5(55.6)$ & $4(44.4)$ & 0.73 \\
Qualitative RV dilation $(n=16)$ & $9(56.3)$ & $7(43.7)$ & 0.41 \\
Qualitative RV dysfunction $(n=8)$ & $5(62.5)$ & $3(37.5)$ & 0.46 \\
\hline
\end{tabular}

$\mathrm{AV}$, arteriovenous; LV, left ventricular; RV, right ventricular.

Table 4. Baseline and echocardiographic findings in patients with qualitative RV dysfunction

\begin{tabular}{|c|c|c|}
\hline \multirow[t]{2}{*}{ Variable } & \multicolumn{2}{|c|}{ Mean value \pm SD or patients, $n(\%)$} \\
\hline & $\begin{array}{l}\text { in patients with RV } \\
\text { dysfunction }(N=8)\end{array}$ & $\begin{array}{l}\text { in patients without RV } \\
\text { dysfunction }(N=65)\end{array}$ \\
\hline Met primary end point, $n(\%)$ & $8(100.0)$ & $39(60.0)$ \\
\hline Time on dialysis, years: mean \pm SD & $3.1 \pm 2.7$ & $2.8 \pm 2.7$ \\
\hline Hypotension, $n(\%)$ & $3(37.5)^{*}$ & $5(7.7)$ \\
\hline \multicolumn{3}{|l|}{ LV size } \\
\hline LVIDd $(\mathrm{mm} \pm \mathrm{SD})$ & $48 \pm 3.1$ & $51 \pm 8.9$ \\
\hline \multicolumn{3}{|l|}{ LV function, $n(\%)$} \\
\hline LV ejection fraction $<50 \%$ & $1(12.5)$ & $6(9.2)$ \\
\hline \multicolumn{3}{|l|}{ RV size } \\
\hline $\mathrm{RV}$ basal diameter $(\mathrm{mm} \pm \mathrm{SD})$ & $48 \pm 4.6^{\dagger}$ & $38 \pm 6.7$ \\
\hline $\mathrm{RV}$ mid-cavity diameter $(\mathrm{mm} \pm \mathrm{SD})$ & $33 \pm 5.7$ & $26 \pm 6.1$ \\
\hline $\mathrm{RV}$ length $(\mathrm{mm} \pm \mathrm{SD})$ & $80 \pm 11.7$ & $76 \pm 9.1$ \\
\hline Qualitative RV dilation, $n(\%)$ & $6(75.0)^{\ddagger}$ & $10(15.4)$ \\
\hline \multicolumn{3}{|l|}{ LA size } \\
\hline LA volume $(\mathrm{mL} \pm \mathrm{SD})$ & $85 \pm 24.5$ & $79 \pm 35.0$ \\
\hline LA AP diameter $(\mathrm{mm} \pm \mathrm{SD})$ & $49 \pm 8.7$ & $43 \pm 7.1$ \\
\hline $\mathrm{RA}$ area $\left(\mathrm{cm}^{2} \pm \mathrm{SD}\right)$ & $24 \pm 10.5$ & $18 \pm 6.5$ \\
\hline $\operatorname{RVSP}(\mathrm{mm} \mathrm{Hg} \pm \mathrm{SD})$ & $34 \pm 12.3$ & $30 \pm 9.1$ \\
\hline Moderate or greater tricuspid regurgitation, $n(\%)$ & $1(12.5)^{\#}$ & $1(1.5)$ \\
\hline
\end{tabular}

SD, standard deviation; LVIDd, left ventricular internal diameter at end-diastole; LV, left ventriclular; RV, right ventricular; AP, antero-posterior; LA, left atrial; RA, right atrial; RVSP, right ventricular systolic pressure. ${ }^{*} p=0.01$ for difference between patients who did and did not have RV dysfunction. ${ }^{\dagger} p=0.02$ for difference. ${ }^{\ddagger} p=0.0001$ for difference. ${ }^{*} p=0.04$ for difference.

20 patients $(25.0 \%)$ had delayed graft function, 44 patients $(55.0 \%)$ had renal failure, and 34 patients $(42.5 \%)$ died.

There were 10 patients with preoperative hypotension, and all of them (100\%) met the primary composite end point, as compared to $57.5 \%$ (23/40) of normotensive patients and $66.7 \%(18 / 27)$ of hypertensive patients $(p=$ 0.039 for difference). Longer dialysis duration prior to transplant was also associated with a higher risk of the primary end point (mean dialysis duration was $3.3 \pm 2.4$ years for those meeting the end point vs. $1.8 \pm 2.7$ years for others, $p=0.016$ ).

\section{Echocardiographic Parameters}

Echocardiographic findings are presented in Table 2. In our population, the average left ventricular internal diameter at end-diastole (LVIDd) was $51 \pm 7.7 \mathrm{~mm}$, and 9 patients $(11.3 \%)$ had LVEF $<50 \%$. The mean RV basal 
Fig. 1. Kaplan-Meier survival analyses by quartile of RV basal diameter. a Time to allcause graft failure. b Time to death. RV, right ventricular.

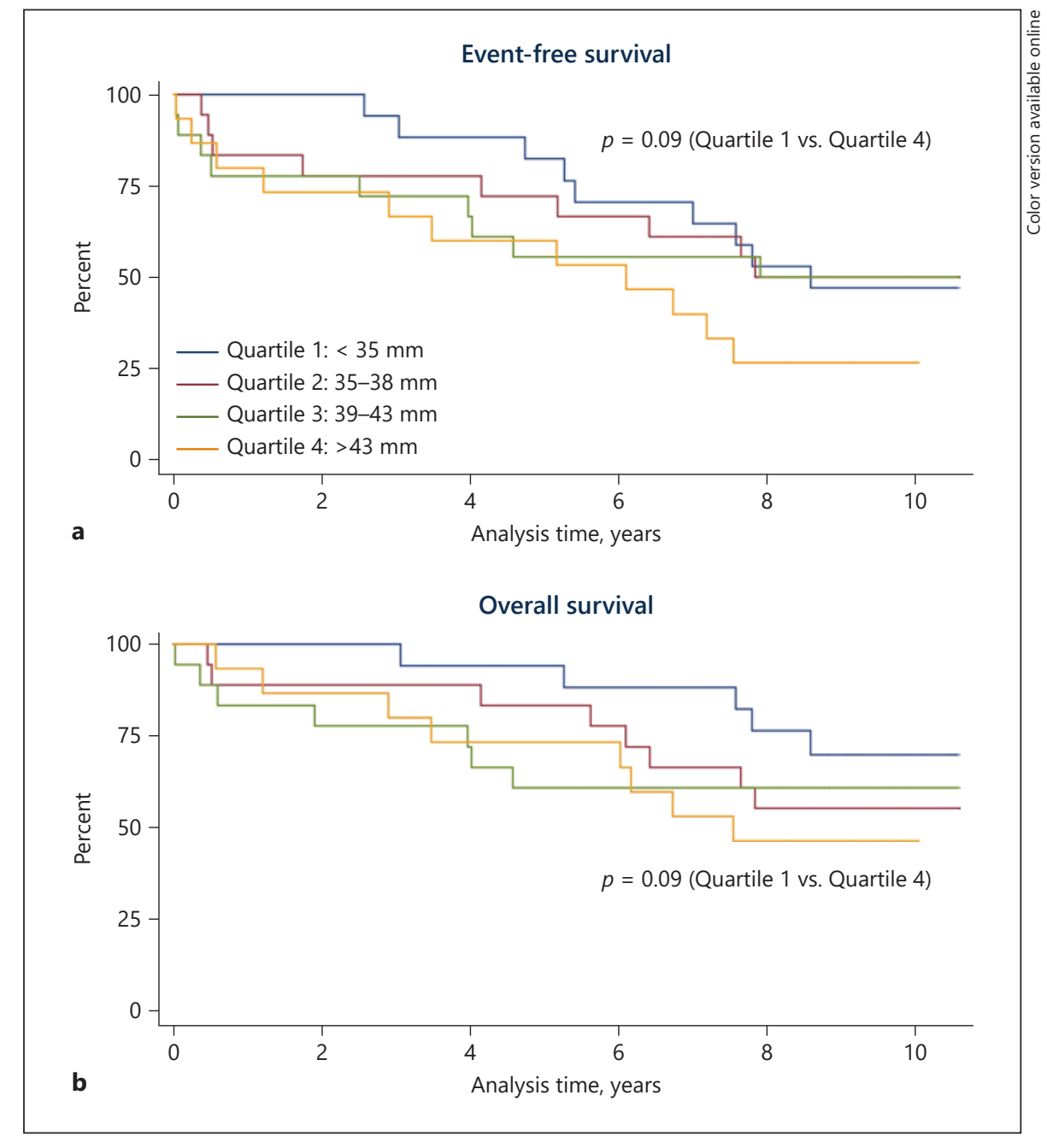

diameter was $39 \pm 7.3 \mathrm{~mm}$, RV mid-cavity diameter was $27 \pm 6.3 \mathrm{~mm}$, and RV length was $76 \pm 9.2 \mathrm{~mm}$. The average right atrial area was $18 \pm 7.0 \mathrm{~cm}^{2}$. Seven patients $(8.8 \%)$ had $E / e^{\prime} \geq 15$. Eight patients $(10 \%)$ had qualitative RV dysfunction and 16 patients (20\%) had qualitative RV dilation. The presence of an arteriovenous (AV) fistula was not significantly associated with LV dilation, LV dysfunction, RV dilation, or RV dysfunction (Table 3). Duration of dialysis was not significantly associated with RV dysfunction or RV dilation ( $p=0.76$ and $p=0.79$, respectively).

RV function could be assessed qualitatively in 73/80 patients (91.3\%). Qualitative RV dysfunction was associated with a significantly higher risk of the primary end point $(p=0.026)$. All 8 patients $(100 \%)$ with qualitative $\mathrm{RV}$ dysfunction met the primary end point, while 39/65 patients $(60.0 \%)$ without RV dysfunction met the end point. Of the patients with RV dysfunction, 3 patients had

Right Ventricular Dysfunction and Renal Transplant Outcomes delayed graft function, 6 patients developed graft failure, and 5 patients died. Among patients with RV dysfunction, $1 / 8$ patients $(12.5 \%)$ had LV dysfunction as well, as compared with $6 / 65$ patients without RV dysfunction (9.2\%, $p=0.78$ for comparison). Patients with RV dysfunction had significantly more dilated RVs than those without RV dysfunction (mean RV basal diameter 48 vs. $38 \mathrm{~mm}, p=0.02$ ). Mean RA area was larger among patients with RV dysfunction, but this difference was not statistically significant ( 24 vs. $18 \mathrm{~cm}^{2}, p=0.25$ ). Preoperative hypotension was more common among patients with RV dysfunction ( $3 / 8$ patients, $37.5 \%$, vs. $5 / 65$ patients, $7.7 \%$ of patients without RV dysfunction, $p=0.01$; Table 4).

RV basal diameter was measurable in $68 / 80$ patients (85.0\%). Patients in the highest quartile of RV basal diameter had a shorter time to all-cause graft failure and death compared to patients in the lowest quartile, though these 
Fig. 2. Kaplan-Meier survival analyses in patients with and without qualitative RV dilation. a Time to all-cause graft failure. $\mathbf{b}$ Time to death. RV, right ventricular.

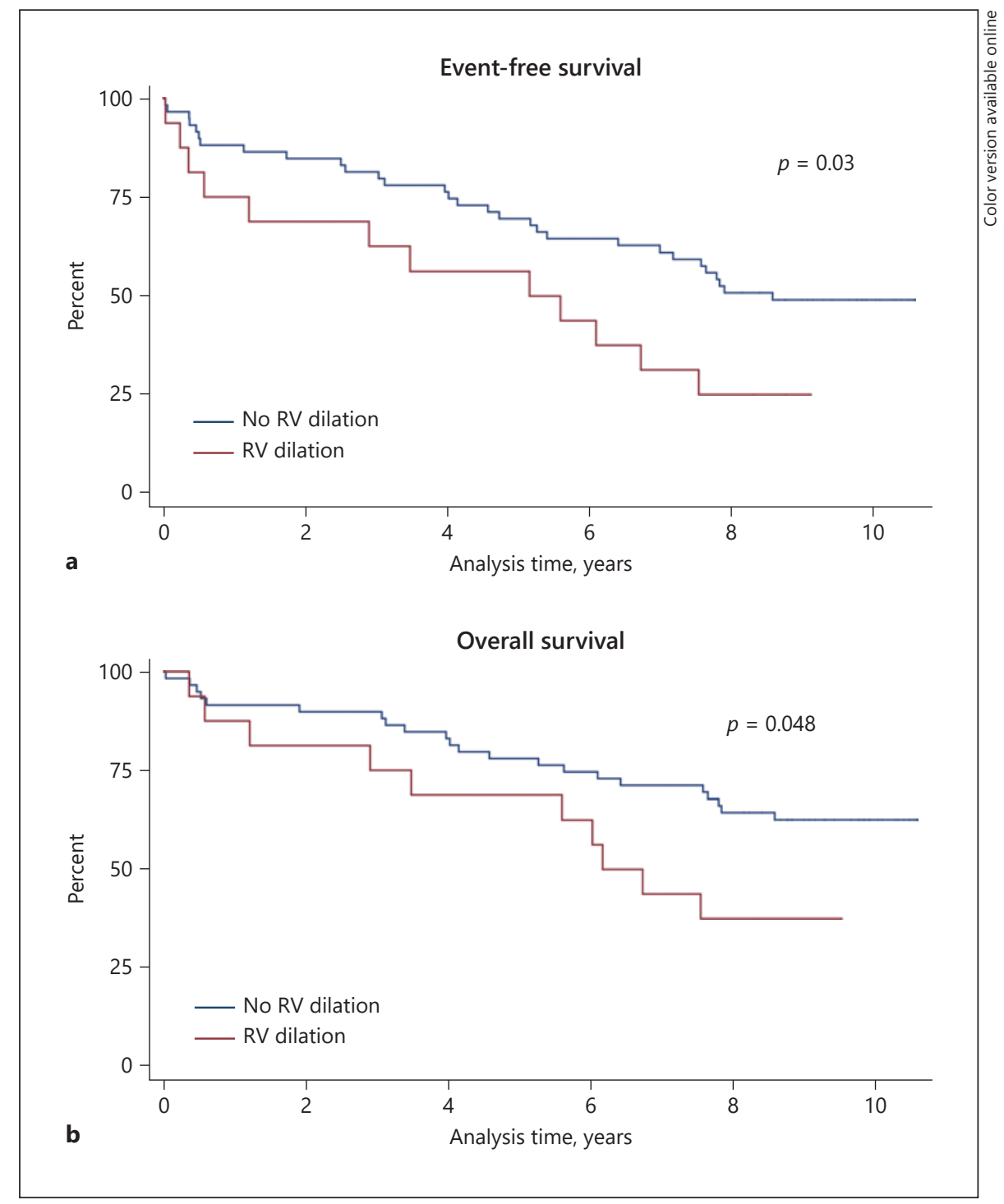

differences did not meet statistical significance $(p=0.09$ for both; Fig. 1). RV dilation could be assessed qualitatively in 75/80 patients (93.8\%). Qualitative RV dilation was associated with a significantly shorter time to allcause graft failure $(p=0.03)$ as well as a significantly shorter time to death ( $p=0.048)$ (Fig. 2).

Notably, RVSP based on tricuspid regurgitation velocity was not possible to be measured in 45/80 (56\%) of patients. RVSP did not differ significantly in patients who did and did not meet the primary end point (mean $32 \pm$ 9.5 vs. $30 \pm 9.5 \mathrm{~mm} \mathrm{Hg}, p=0.39$ ). RVSP was moderately correlated with RV basal diameter $(r=0.57, p=0.001)$ (Fig. 3). The prevalence of moderate or greater tricuspid regurgitation in our study was $2.5 \%$.

\section{Discussion}

A number of echocardiographic studies have demonstrated that the prevalence of $\mathrm{PH}$ is high among patients with ESRD [12-14]. There are many potential causes for the increased prevalence in this population, including left ventricular systolic and/or diastolic dysfunction, chronic volume overload due to inadequate ultrafiltration, high cardiac output in the setting of AV fistula and/or chronic anemia, and sleep apnea [15]. Interestingly, in a study of 76 patients with ESRD, Abdelwhab and Elshinnawy [13] demonstrated that the prevalence of $\mathrm{PH}$ (defined as an estimated RVSP of $>35 \mathrm{~mm} \mathrm{Hg}$ on TTE) was high regardless of whether the patients were managed with hemodi- 
Fig. 3. Correlation of RVSP with RV basal diameter. RVSP, right ventricular systolic pressure; RV, right ventricular.

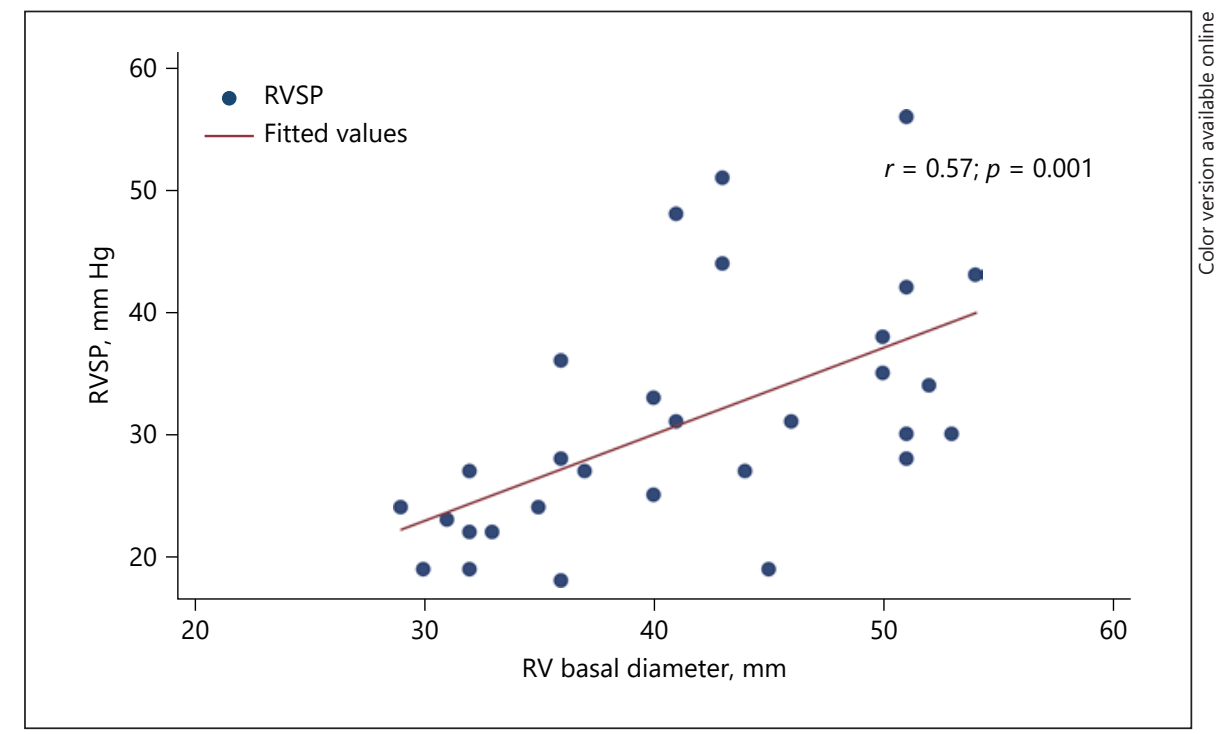

alysis (44.4\%) or had not yet initiated dialysis and were managed with medications alone (32.3\%).

Multiple studies have shown the presence of $\mathrm{PH}$ to be a strong prognostic indicator in the ESRD and posttransplant populations. Stallworthy et al. [16] performed a retrospective review of 739 patients evaluated for renal transplantation and found that $\mathrm{PH}$ and/or RV dysfunction was significantly associated with all-cause mortality (hazard ratio 1.91, $p=0.001$ ). Zlotnick et al. [17] showed that RVSP $\geq 35 \mathrm{~mm} \mathrm{Hg}$ was associated with early graft failure in deceased donor recipients ( $43 \%$ in patients with elevated RVSP vs. $6 \%$ in those with normal RVSP, $p=$ 0.002). Issa et al. [18] found that a markedly elevated RVSP of $>50 \mathrm{~mm} \mathrm{Hg}$ was associated with reduced survival post-transplantation $(p=0.06)$. However, RVSP could not be measured in a large number of patients (36/94 [38.3\%] for Zlotnick et al. [17] and 109/324 [33.6\%] for Issa et al. [18]). Finally, Caughey et al. [19] considered that left atrial pressure might be associated with outcomes in patients with $\mathrm{PH}$ who underwent kidney transplantation. They found that 5-year mortality was higher in patients with $\mathrm{PH}$ in the absence of elevated left atrial pressure, suggesting that patients with significant pulmonary vascular disease are at highest risk [19].

$\mathrm{RV}$ dilation and dysfunction are strong prognostic indicators in $\mathrm{PH}$ [8], and among patients beginning hemodialysis, RV dysfunction has been shown to be independently associated with mortality [20]. Cardiovascular-related hospitalizations and events occur more frequently after a long interdialytic interval [21]. Tsilonis et al. [22] measured the echocardiographic parameters of LV and RV function at the end of 3- and 2-day interdialytic intervals. They found that while LV systolic function remains unchanged, there was a significant increase in inferior vena cava diameter, RA volume, and RVSP during the 3-day interval, suggesting that right heart failure is a major driver of poor outcomes. They hypothesized that unlike the LV, which has greater wall thickness, the RV is highly susceptible to volume overload [22]. Furthermore, hemodialysis involves large and frequent fluid shifts. RV dysfunction has been associated with intradialytic hypotension, which results in myocardial stunning and ischemia [23]. While we found that duration of dialysis was not significantly associated with development of RV dysfunction or RV dilation, this may be a consequence of evaluating a small study population. Interestingly, while having an AV fistula is a recognized risk factor for high-output heart failure, having an AV fistula was not associated with a significantly higher rate of LV dilation, LV dysfunction, RV dilation, or RV dysfunction in our small study [24]. This may also be a consequence of evaluating a small study population.

The goal of our study was to assess whether markers of $\mathrm{RV}$ dilation and dysfunction are associated with poor outcomes following renal transplant, including delayed graft function, graft failure, and all-cause mortality. Particularly, important findings include the high prevalence of RV dysfunction and dilation in this population (10 and $20 \%$, respectively) and the fact that patients with qualitative RV dysfunction had a significantly higher likelihood of reaching the primary end point. As discussed above, while there are many potential causes of RV dysfunction in patients with ESRD, in our study population the exact 

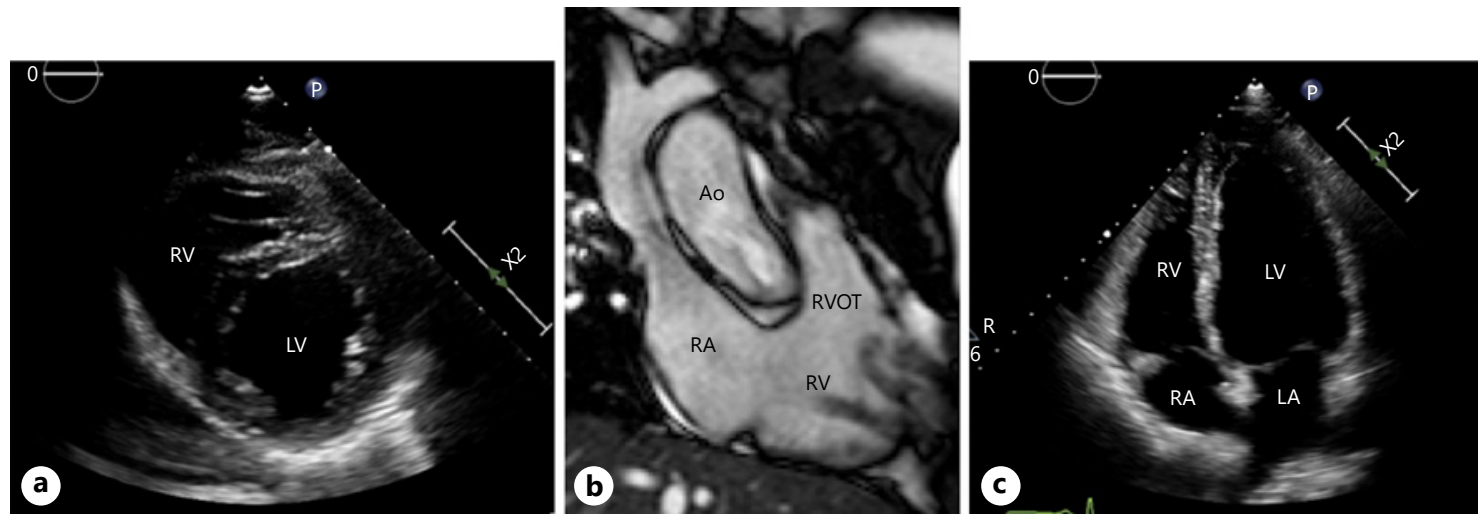

Fig. 4. Complex shape of the RV. a In this parasternal short-axis view on TTE, the RV appears to be a crescent wrapped around the LV. $\mathbf{b}$ In this RV 3-chamber view on cardiovascular magnetic resonance, the RV and RVOT appear like a baseball glove gripping the Ao. RA. c In this apical 4-chamber view on TTE, the RV looks like a triangle or sail. This is perhaps the most important view for assessment of RV size and function on TTE. LA, left atrium; RV, right ventricle; TTE, transthoracic echocardiography; LV, left ventricle; RVOT, right ventricular outflow tract; Ao, aorta; RA, right atrium.

causes of RV dysfunction were not known. Furthermore, $\mathrm{RV}$ dilation was associated with shorter time to all-cause graft failure and shorter time to death. There are multiple mechanisms by which RV dilation and dysfunction may contribute to graft failure, including venous congestion and low cardiac output. A retrospective review of renal transplant recipients with graft failure found the proportion of allograft dysfunction due to cardiorenal syndrome to be $4.6 \%$, suggesting that venous congestion is an important cause of allograft dysfunction [25]. Functional tricuspid regurgitation in the setting of $\mathrm{RV}$ dilation and dysfunction can further increase venous pressure and potentially affect renal perfusion pressure. Hypotension as a result of autonomic dysfunction, vascular calcification, and low cardiac output could be contributing to graft failure as well. Additional studies are needed to clarify these mechanisms. Our findings suggest that RV assessment by TTE could be extremely useful in identifying patients who require further cardiovascular optimization and work-up, including diagnostic testing and adjustment of ultrafiltration goals, prior to renal transplantation.

While prior studies in the pretransplant population have focused on RVSP for PH screening, it is important to note that RVSP could not be measured in the majority of patients in our real-world sample. An incomplete or poor-quality tricuspid regurgitation Doppler envelope often precludes RVSP measurement. Common reasons for this include absence of tricuspid regurgitation, eccentricity of the tricuspid regurgitation jet, and patient obesity. While an elevated RVSP should raise suspicion for
$\mathrm{PH}$, an echocardiogram report that omits RVSP or includes a "normal" RVSP based on a poor-quality signal should not be considered reassuring. RVSP and RV basal diameter were moderately correlated in our population.

When a TTE is ordered for a pretransplant patient, we recommend that the ordering physician specifically request detailed comments on RV size and function. Because of the right ventricle's unique shape - conceptualized as a pyramid, crescent, or baseball glove, depending upon one's perspective (Fig. 4) - it is very difficult to model geometrically. No single linear or 2-dimensional parameter can adequately determine whether the right ventricle is dilated or dysfunctional. Assessment of RV size in multiple echocardiographic views and in relation to left ventricular size is critically important. As such, assessment of the right ventricle by a thoughtful and experienced echocardiographer is highly desirable.

\section{Limitations}

This was a retrospective, single-center study evaluating a relatively small population of patients. RV dimensions and function are volume-dependent, so the timing of each echocardiogram in relation to dialysis could have impacted the results. At the time these TTEs were obtained, tricuspid annular plane systolic excursion (a Mmode echocardiographic parameter) and tricuspid annular S' velocity (a tissue Doppler parameter) were not standard at our institution, although they are recommended for RV function assessment per current guidelines [10]. Finally, in an effort to emulate real-world practice, we 
chose not to exclude any patients with limited TTE image quality, though individual data points were omitted when accurate measurements could not be made.

\section{Conclusions}

In this retrospective study, we have shown that RV dilation and dysfunction on pretransplant TTE are associated with adverse outcomes following renal transplant, including delayed graft function, graft failure, and allcause mortality. Assessment of RV size and function by TTE could be invaluable in risk stratification prior to renal transplantation. Large, prospective, multicenter studies are needed to corroborate these findings.

\section{Statement of Ethics}

The University of Michigan institutional review board approved the retrospective study as part of a multivariate analysis of outcomes of the University of Michigan transplantation program.

\section{Conflict of Interest Statement}

Megan Joseph: None. Francis Tinney: None. Abhijit Naik: None. Raviprasenna Parasuraman: None. Milagros SamaniegoPicota: None. Nicole Bhave: None.

\section{Funding Sources}

There was no specific funding for this project.

\section{Author Contributions}

Megan Joseph, MD, participated in the writing of the paper, performance of the research, and data analysis. Francis Tinney, $\mathrm{MD}$, participated in the performance of the research and data analysis. Abhijit Naik, MD, participated in the writing of the paper and data analysis. Raviprasenna Parasuraman, MD, participated in the writing of the paper and data analysis. Milagros Samaniego-Picota, $\mathrm{MD}$, participated in the writing of the paper and data analysis. Nicole Bhave, MD, participated in research design, echocardiographic analysis, writing of the paper, and data analysis.

\section{References}

1 Glicklich D, Vohra P. Cardiovascular risk assessment before and after kidney transplantation. Cardiol Rev. 2014;22(4):153-62.

2 Lentine KL, Costa SP, Weir MR, Robb JF, Fleisher LA, Kasiske BL, et al. Cardiac disease evaluation and management among kidney and liver transplantation candidates: a scientific statement from the American Heart Association and the American College of cardiology foundation. J Am Coll Cardiol. 2012; 60(5):434-80.

3 Collins AJ, Foley RN, Chavers B, Gilbertson D, Herzog C, Ishani A, et al. US renal data system 2013 annual data report. Am J Kidney Dis. 2014;63(1 Suppl):A7.

4 Bolignano D, Pisano A, Coppolino G, Tripepi GL, D'Arrigo G. Pulmonary hypertension predicts adverse outcomes in renal patients: a systematic review and meta-analysis. Ther Apher Dial. 2019;23(4):369-84.

5 Rangaswami J, Bhalla V, Blair JEA, Chang TI, Costa S, Lentine KL, et al. Cardiorenal syndrome: classification, pathophysiology, diagnosis, and treatment strategies: a scientific statement from the American Heart Association. Circulation. 2019;139(16):e840-e78.

6 Lentine KL, Villines TC, Axelrod D, Kaviratne S, Weir MR, Costa SP. Evaluation and management of pulmonary hypertension in kidney transplant candidates and recipients: concepts and controversies. Transplantation. 2017;101(1):166-81.
7 McLaughlin VV, Shah SJ, Souza R, Humbert M. Management of pulmonary arterial hypertension. J Am Coll Cardiol. 2015;65(18): 1976-97.

8 Baggen VJ, Leiner T, Post MC, van Dijk AP, Roos-Hesselink JW, Boersma E, et al. Cardiac magnetic resonance findings predicting mortality in patients with pulmonary arterial hypertension: a systematic review and metaanalysis. Eur Radiol. 2016;26(11):3771-80.

9 Lang RM, Bierig M, Devereux RB, Flachskampf FA, Foster E, Pellikka PA, et al. Recommendations for chamber quantification: a report from the American Society of echocardiography's guidelines and standards committee and the chamber quantification writing group, developed in conjunction with the European Association of echocardiography, a branch of the European Society of cardiology. J Am Soc Echocardiogr. 2005;18(12): 1440-63.

10 Lang RM, Badano LP, Mor-Avi V, Afilalo J, Armstrong A, Ernande L, et al. Recommendations for cardiac chamber quantification by echocardiography in adults: an update from the American Society of Echocardiography and the European Association of cardiovascular imaging. J Am Soc Echocardiogr. 2015; 28(1):1-39.e14
11 Zoghbi WA, Adams D, Bonow RO, EnriquezSarano M, Foster E, Grayburn PA, et al. Recommendations for noninvasive evaluation of native valvular regurgitation: a report from the American Society of echocardiography developed in collaboration with the society for cardiovascular magnetic resonance. J Am Soc Echocardiogr. 2017;30(4):303-71.

12 Yigla M, Nakhoul F, Sabag A, Tov N, Gorevich B, Abassi Z, et al. Pulmonary hypertension in patients with end-stage renal disease. Chest. 2003;123(5):1577-82.

13 Abdelwhab S, Elshinnawy S. Pulmonary hypertension in chronic renal failure patients. Am J Nephrol. 2008;28(6):990-7.

14 Nakhoul F, Yigla M, Gilman R, Reisner SA, Abassi Z. The pathogenesis of pulmonary hypertension in haemodialysis patients via arterio-venous access. Nephrol Dial Transplant. 2005;20(8):1686-92.

15 Bolignano D, Rastelli S, Agarwal R, Fliser D, Massy Z, Ortiz A, et al. Pulmonary hypertension in CKD. Am J Kidney Dis. 2013;61(4): 612-22.

16 Stallworthy EJ, Pilmore HL, Webster MW, Sidhu KK, Curry EM, Brown P, et al. Do echocardiographic parameters predict mortality in patients with end-stage renal disease? Transplantation. 2013;95(10):1225-32. 
17 Zlotnick DM, Axelrod DA, Chobanian MC, Friedman S, Brown J, Catherwood E, et al. Non-invasive detection of pulmonary hypertension prior to renal transplantation is a predictor of increased risk for early graft dysfunction. Nephrol Dial Transplant. 2010; 25(9):3090-6.

18 Issa N, Krowka MJ, Griffin MD, Hickson LJ, Stegall MD, Cosio FG. Pulmonary hypertension is associated with reduced patient survival after kidney transplantation. Transplantation. 2008;86(10):1384-8.

19 Caughey MC, Detwiler RK, Sivak JA, RoseJones LJ, Kshirsagar AV, Hinderliter AL Fiveyear outcomes of pulmonary hypertension with and without elevated left atrial pressure in patients evaluated for kidney transplantation. Transplantation. 2020 Oct; 104(10): 2113-9.

20 Hickson LJ, Negrotto SM, Onuigbo M, Scott CG, Rule AD, Norby SM, et al. Echocardiography criteria for structural heart disease in patients with end-stage renal disease initiating hemodialysis. J Am Coll Cardiol. 2016; 67(10):1173-82.

21 Foley RN, Gilbertson DT, Murray T, Collins AJ. Long interdialytic interval and mortality among patients receiving hemodialysis. $\mathrm{N}$ Engl J Med. 2011;365(12):1099-107.

22 Tsilonis K, Sarafidis PA, Kamperidis V, Loutradis C, Georgianos PI, Imprialos K, et al. Echocardiographic parameters during long and short interdialytic intervals in hemodialysis patients. Am J Kidney Dis. 2016;68(5): $772-81$.

23 Sun M, Cao X, Guo Y, Tan X, Dong L, Pan C, et al. Long-term impacts of hemodialysis on the right ventricle: assessment via 3-dimensional speckle-tracking echocardiography. Clin Cardiol. 2018;41(1):87-95.

24 Samarendra P, Ramkumar M, Sharma V, Kumari S. Cardiorenal syndrome in renal transplant recipients: It's the fistula at fault: a case series. Clin Transplant. 2018;32(11):e13417.

25 Waiser J, Knebel F, Rudolph B, Wu K, Müller E, Sanad W, et al. Renal allograft loss caused by cardiorenal syndrome: frequency and diagnosis. Transplantation. 2015;99(6):1208-15. 